\title{
Kundenbindung von Noncomplainern: \\ Neue Aspekte des Beschwerdemanagement
}

\author{
Christian Brock \\ Jun.-Prof. für Distanzhandel und Service Marketing \\ Zeppelin Universität \\ Am Seemooser Horn 20 \\ 88045 Friedrichshafen
}

Markus Blut

Habilitand am Lehrstuhl für Marketing

Technische Universität Dortmund

Otto-Hahn-Straße 6

44221 Dortmund

\author{
Heiner Evanschitzky \\ Professor für Marketing \\ Department of Marketing \\ University of Strathclyde \\ 173 Cathedral Street \\ G4 0RQ Glasgow, UK \\ Martin Ahlert \\ Geschäftsführer des F\&C \\ Westfälische Wilhelms-Universität Münster \\ Fliednerstraße 21 \\ 48149 Münster \\ Peter Kenning \\ Professor für Marketing \\ Zeppelin Universität \\ Am Seemooser Horn 20 \\ 88045 Friedrichshafen
}

Beitrag eingereicht zum ZfB Sonderheft „Kundenmanagement“ 
Dr. Christian Brock ist Jun.-Prof. für Distanzhandel und Service Marketing an der Zeppelin Universität, Am Seemooser Horn 20, 88045 Friedrichshafen, Telefon: +49-(0)75416009-1291 E-Mail: christian.brock@zeppelin-university.de

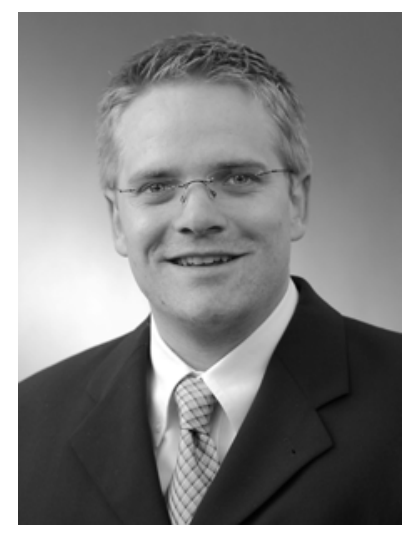

Dr. Markus Blut ist Habilitand am Lehrstuhl für Marketing der Technischen Universität Dortmund, Otto-Hahn-Strasse 6, 44221 Dortmund, Telefon: +49-(0)231-755-3277, E-Mail: Markus.Blut@tu-dortmund.de.

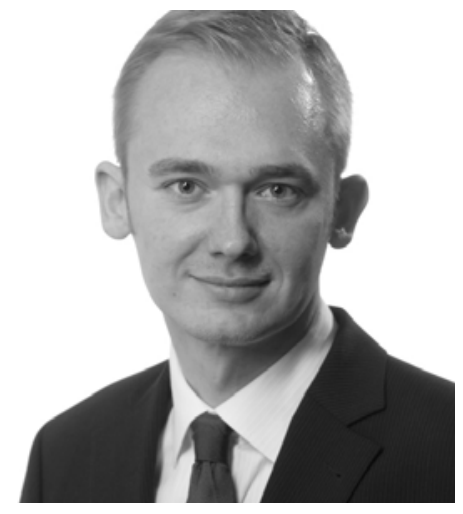

Prof. Dr. Heiner Evanschitzky ist Professor für Marketing an der University of Strathclyde, United Kingdom, 173 Cathedral Street, Glasgow G4 0RQ, E-Mail: Evanschitzky@strath.ac.uk

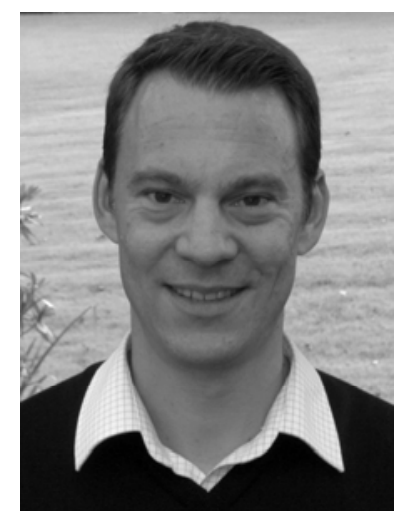


Dr. Martin Ahlert ist Geschäftsführer des Internationalen Centrums für Franchising \& Cooperation (F\&C) an der Westfälischen Wilhelms-Universität Münster, Fliednerstr. 21, 48149 Münster, Tel.: +49-(0)251-83-29953, E-Mail: Ahlert.Martin@t-online.de.

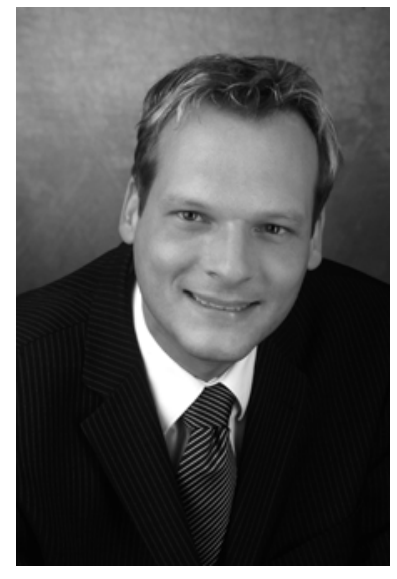

Prof. Dr. Peter Kenning ist Professor für Marketing an der Zeppelin Universität, Am Seemooser Horn 20, 88045 Friedrichshafen; E-Mail: Peter.Kenning@zeppelin-university.de

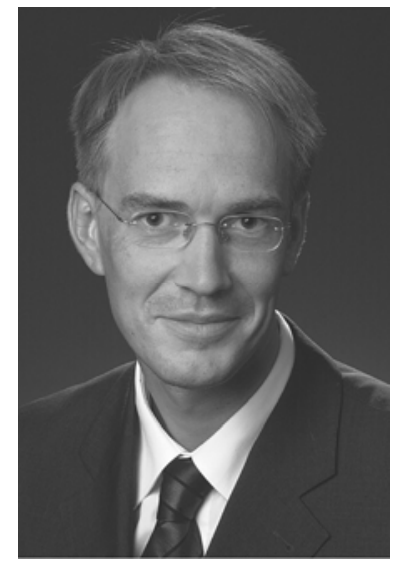




\section{Kundenbindung von Noncomplainern: \\ Neue Aspekte des Beschwerdemanagement}

\section{Überblick}

- Gegenstand des vorliegenden Beitrags sind Kunden, die trotz eines negativen Vorfalls bei der Leistungserbringung ihre Unzufriedenheit nicht gegenüber dem Anbieter artikulieren („Noncomplainer“). Diese Kundengruppe fand in der bisherigen Beschwerdeforschung nur wenig Beachtung, obwohl verschiedene Studien nachweisen konnten, dass nur ein sehr geringer Anteil der unzufriedenen Kunden ihre Beschwerde überhaupt gegenüber dem Unternehmen artikuliert.

- Die vorliegende Studie leistet einen Beitrag zu einem besseren Verständnis über (1) die Wirkung von negativen Vorfällen auf die Kundenbindung und (2) die Rolle von Wiedergutmachungen aus Sicht der Kunden. Hierdurch lassen sich (3) Rückschlüsse für die Beschwerdestimulierung von Noncomplainern ziehen. Hierzu vergleichen die Autoren des Beitrags Noncomplainer mit Kunden, die eine Wiedergutmachung erfahren haben, und solchen ohne negativen Vorfall. Dabei konzeptualisieren sie die Kundenbindung umfassend und werten erstmals sowohl Kundenbefragungs- als auch Transaktionsdaten nach einem negativen Vorfall aus.

Stichworte: Kundenmanagement, Beschwerdemanagement, Beschwerdestimulierung, Noncomplainer 


\title{
Zusammenfassung
}

Gegenstand des vorliegenden Beitrags sind Kunden, die trotz eines erlebten negativen Vorfalls bei der Leistungserbringung ihre Unzufriedenheit nicht gegenüber dem Anbieter artikulieren (,Noncomplainer"). Diese Kundengruppe fand in der bisherigen Beschwerdeforschung nur wenig Beachtung, obwohl einzelne Untersuchungen nachweisen konnten, dass nur ein sehr geringer Anteil der unzufriedenen Kunden ihre Beschwerde überhaupt gegenüber dem Unternehmen artikuliert. Ziel des vorliegenden Beitrags ist es daher, (1) die Wirkung von negativen Vorfällen auf Kundenbindung zu untersuchen und (2) die Rolle von Wiedergutmachungen aus Sicht der Kunden besser zu verstehen. Hierdurch können (3) Rückschlüsse für die Beschwerdestimulierung von Noncomplainern gezogen werden.

\begin{abstract}
This paper provides a comprehensive assessment of customers who experience a service failure and do not voice their dissatisfaction to the provider ('non-complainer'). This group of customers has been largely neglected by complaint research; although first studies indicate that only a small percentage of customers being dissatisfied voice their complaint to the firm. To address this issue, this research intends to (1) examine the impact of negative critical incidents on customer loyalty and (2) contribute to a better understanding of the role of customer recovery. Based on these analyses, (3) the relevance of complaint stimulation is derived. In doing so, management receives valuable information on how to deal with noncomplainers.
\end{abstract}




\section{Einleitung}

Das Phänomen der Kundenbeschwerde beschäftigt sowohl die Marketingforschung als auch die Kundenmanagementpraxis seit mehreren Jahrzehnten (vgl. bspw. Bearden/Oliver 1985; Singh 1990a, b; Blodgett/Granbois/Walters 1993; Tax/Brown/Chundrashekaran 1998; Maxham 2001; Davidow 2003; Homburg/Fürst 2005, 2007; Mittal/Huppertz/Khare 2008). Die meisten Veröffentlichungen in diesem Bereich betrachten vornehmlich das Verhalten der Kunden, die sich aufgrund eines negativen Vorfalls im Rahmen der Leistungserbringung gegenüber dem Unternehmen beschweren. In diesem Zusammenhang wurde insbesondere der angemessene unternehmensseitige Umgang mit dieser Kundengruppe den sog. Complainern diskutiert (vgl. Homburg/Fürst 2005, 2007; Smith/Bolton 2002; Tax et al. 1998). Neben dieser Kundengruppe gibt es jedoch auch Kunden, die ihre Unzufriedenheit nach einem negativen Vorfall nicht gegenüber dem Unternehmen artikulieren, die sog. Gruppe der Noncomplainer (vgl. Bendapudi/Berry 1997; Ganesh/Arnold/Reynolds 2000; Jones/Sasser 1995; Keaveney 1995; Richins 1987; Singh 1990a; Voorhees/Brady/Horowitz 2006; TARP 1986) - diese ist Gegenstand des vorliegenden Beitrags.

Bisherige Studien zum Kundentypus des Noncomplainer versuchen zumeist, eine Art Profil des Noncomplainers zu erstellen, um diese besser identifizieren zu können (vgl. bspw. Homburg/Hoyer/Stock-Homburg 2007; Pick 2008; Rutsatz 2004; Tokman/Davis/Lemon 2006). Die explizite Betrachtung des Kundenbindungsniveaus von Noncomplainern ist dagegen Gegenstand nur weniger Studien. Voorhees/Brady/Horowitz (2006, S. 514) betonen in diesem Zusammenhang: „Unfortunately, very little research exists that specifically investigated noncomplainers or compared noncomplainers with other customer groups across important outcome measures." In ihrem Beitrag zeigen die Autoren die Bedeutung von Noncomplainern auf Basis der von ihnen geäußerten Wiederkaufabsicht, der negativen Gefühle aufgrund des Vorfalls und der negativer Kommunikation gegenüber Dritten auf. Mit der Betrachtung der Wiederkaufabsicht wird jedoch lediglich eine Facette der Kundenbindung adressiert. Ohne ein differenziertes Verständnis über das Bindungsniveau der Kunden sind Implikationen zur Beschwerdestimulierung und Rückgewinnung einzelner Kundengruppen nur schwerlich möglich. So zeigen De Matos/Henrique/Rossi (2007) in ihrem Beitrag, dass die Beschwerdebearbeitung zwar eine Verbesserung der Kundeneinstellung bewirkt, die Verhaltenabsichten ändern sich dagegen nicht wie erwartet. Eine Betrachtung der Wirkung auf das tatsächliche Kaufverhalten der Kunden würde weiteren Aufschluss über die Relevanz der Beschwerdestimulierung von Noncomplainern geben. 
Dementsprechend folgt der vorliegende Beitrag der Empfehlung von De Matos/Henrique/Rossi (2007) und untersucht das Kundenbindungsniveau der Noncomplainer anhand des vierstufigen Kundenbindungsmodells von Oliver (1997, 1999). Durch die Differenzierung zwischen kognitiver, affektiver, konativer und aktionaler Kundenbindung erwarten wir ein besseres Verständnis über die Wirkung von negativen Vorfällen auf die Kundenbindung zu erlangen. Ferner kann die Wirkung von Wiedergutmachungen besser beurteilt werden und Rückschlüsse auf die Relevanz der Beschwerdestimulierung von Noncomplainern gezogen werden.

Die praktische Bedeutung der Thematik lässt sich an folgendem Beispiel veranschaulichen: Im Jahre 2005 wurden bei der Festnetzsparte T-Com der Deutschen Telekom AG rund 50.000 Kundenbeschwerden pro Woche registriert. Dies entspricht in etwa 2,6 Millionen Kundenbeschwerden pro Jahr. Bei einem Kundenstamm von rund 35 Millionen liegt die Quote der negativen Vorfälle, die zur Beschwerdeartikulation geführt haben, somit bei ca. 7\% [1]. Einzelne Studien argumentieren, dass lediglich 5-10\% der Kunden ihre Unzufriedenheit gegenüber dem Unternehmen äußern (was selbstredend eine branchenabhängige Größe ist und nicht pauschal über alle Branchen hinweg gilt). Rechnet man im Falle der Telekom mit einer sehr konservativen Noncomplainer-Quote von 70\% entspräche dies etwa 6 Millionen Noncomplainern, die im Jahre 2005 ihre Beschwerde nicht geäußert haben. Dies sind rund $17 \%$ aller T-Com Kunden, die potenziell den Anbieter wechseln und durch ein besseres Beschwerdemanagement davon abgehalten werden könnten. Für die Deutschen Telekom AG ist es nun wichtig zu wissen, (1) ob die Noncomplainer weniger loyal sind als Kunden ohne Vorfall (2) ob durch eine Beschwerdestimulierung und zufrieden stellende Bearbeitung das ursprüngliche Kundenbindungsniveau wiederhergestellt werden kann und (3) ob die ergriffenen Maßnahmen lediglich die Einstellungen der Kunden verbessern oder aber, ob auch die Verhaltensabsichten und - noch wichtiger - das tatsächliche Kaufverhalten sich im Sinne des Unternehmens entwickeln. Vor diesem Hintergrund lautet die übergeordnete Forschungsfrage des vorliegenden Beitrags wie folgt:

Welches Bindungsniveau weisen Noncomplainer nach einem negativen Vorfall gegenüber dem betreffenden Unternehmen im Vergleich zu anderen Kundengruppen auf?

Es gilt zu überprüfen, inwiefern sich Noncomplainer hinsichtlich ihrer Bindung gegenüber dem Unternehmen von anderen Kundengruppen mit einem kritischen Vorfall (und 
erfahrener Wiedergutmachung) und einer Kontrollgruppe ohne jeglichen Vorfall unterscheiden. Zur Beantwortung dieser Forschungsfrage wird im Folgenden zunächst ein Überblick über den Stand der Forschung im Bereich des Beschwerdemanagement gegeben. Anschließend werden die Exit-Voice-Loyalty-Theorie sowie die Equity-Theorie vorgestellt, da beide Theorien in der Lage sind, das Beschwerdeverhalten sowohl der Complainer als auch der Noncomplainer zu erklären. Im Anschluss daran wird konfirmatorisch die Relevanz von Noncomplainern untersucht. Hier ist insbesondere die Betrachtung des tatsächlichen Kaufverhaltens nach dem negativen Vorfall explizit hervorzuheben, da somit im Gegensatz zu vielen anderen Studien im Bereich des Beschwerdemanagement, kausale Aussagen über das Verhalten von Noncomplainern nach einem negativen Vorfall getroffen werden können. Abschließend werden die Limitationen dieses Beitrags sowie der sich daraus ergebende weitere Forschungsbedarf diskutiert.

\section{Stand der Forschung zum Beschwerdemanagement}

\subsection{Noncomplainer als Betrachtungsgegenstand}

In den letzten Jahren hat die betriebswirtschaftliche Forschung zahlreiche Einblicke in die Motivlage und das Verhalten von Complainern gewonnen, also der Kunden, die sich nach einer negativen Erfahrung gegenüber dem Unternehmen beschwert haben. Wenig bekannt ist allerdings über die Gruppe der Noncomplainer. Als Grund für dieses Forschungsdefizit ist anzuführen, dass solche Studien großzahlige Befragungen voraussetzen, um ausreichend Kunden in der Stichprobe zu haben, die in der jüngsten Vergangenheit sowohl einen negativen Vorfall erlebt als auch gleichzeitig ihre Beschwerde nicht geäußert haben. Die wenigen Studien, die bislang existieren, betrachten nur ausgewählte Aspekte der Complainer im Vergleich $\mathrm{zu}$ den Noncomplainern bspw. hinsichtlich deren Demographika (Bearden/Oliver 1985; Bolfing 1989; Gronhaug/Zaltmann 1981; Singh 1990b), differenter Persönlichkeitsmerkmale (Bodey/Grace 2006; Bolfing 1989; Gronhaug/Zaltmann 1981), deren Einstellung gegenüber der Beschwerde (Bearden/Oliver 1985; Fornell/Westbrook 1979; Singh 1990b; Voorhees/Brady 2005), deren Einstellung gegenüber dem Unternehmen (Jacoby/Jaccard 1981; Richins 1983b), des Wertes des Beschwerdeobjekts (Shuptrine/Wenglorz 1981; TARP 1979) oder dem Niveau der Unzufriedenheit (Singh/Panday 1990; Singh/Wilken 1996). Ziel dieser Studien ist es zumeist Noncomplainer 
zu identifizieren, indem eine Art Profil dieser Kunden erstellt wird. Demgegenüber vernachlässigen diese Studien die Betrachtung des Bindungsniveaus der Noncomplainer; allenfalls werden einzelne Facetten der Kundenbindung rudimentär betrachtet (Voorhees/Brady/Horowitz 2006). Insbesondere hat keine Studie bisher anhand des realen Kaufverhaltens nach dem negativen Vorfall geprüft, inwiefern die Beschwerdestimulierung aus Unternehmenssicht wünschenswert ist. Dementsprechend widmet sich der folgende Abschnitt zwei Theorien die zur Erklärung des Beschwerdeverhaltens herangezogen werden können.

\subsection{Theoretische Grundlagen: Exit-Voice-Loyalty-Theorie sowie Equity-Theorie}

In der Beschwerdeforschung, insbesondere zur Fundierung und Erklärung der unterschiedlichen Facetten des Beschwerdeverhaltens, existierst keine alleinstehende und alles erklärende Theorie. Blodgett et al. konstatieren vor diesem Hintergrund: „There is no single theory of consumer complaint behavior; rather, the study of complaining behavior is based upon several different theories from various fields of study (Blodgett et al. 1993, S. 402)." Zwei Theorien haben sich jedoch zur Erklärung des differenten Beschwerdeverhaltens unzufriedener Kunden als fundamental erwiesen: Das sind die mikroökonomische Exit-Voice-Loyalty-Theorie von Hirschman (1970) und die EquityTheorie von Adams (1963). Diese bilden auch nachfolgend den theoretischen Rahmen des Beitrags.

Die Exit-Voice-Loyalty-Theorie besteht aus drei Ebenen, im Einzelnen sind dies Branchencharakteristika, individuelle Kundenmerkmale sowie Reaktionen der Kunden nach einem negativen Vorfall (vgl. Abb. 1).

Abb. 1: Darstellung der Exit-Voice-Loyalty-Theorie Quelle: Eigene Darstellung, in Anlehnung an Singh 1990a, S. 2. 


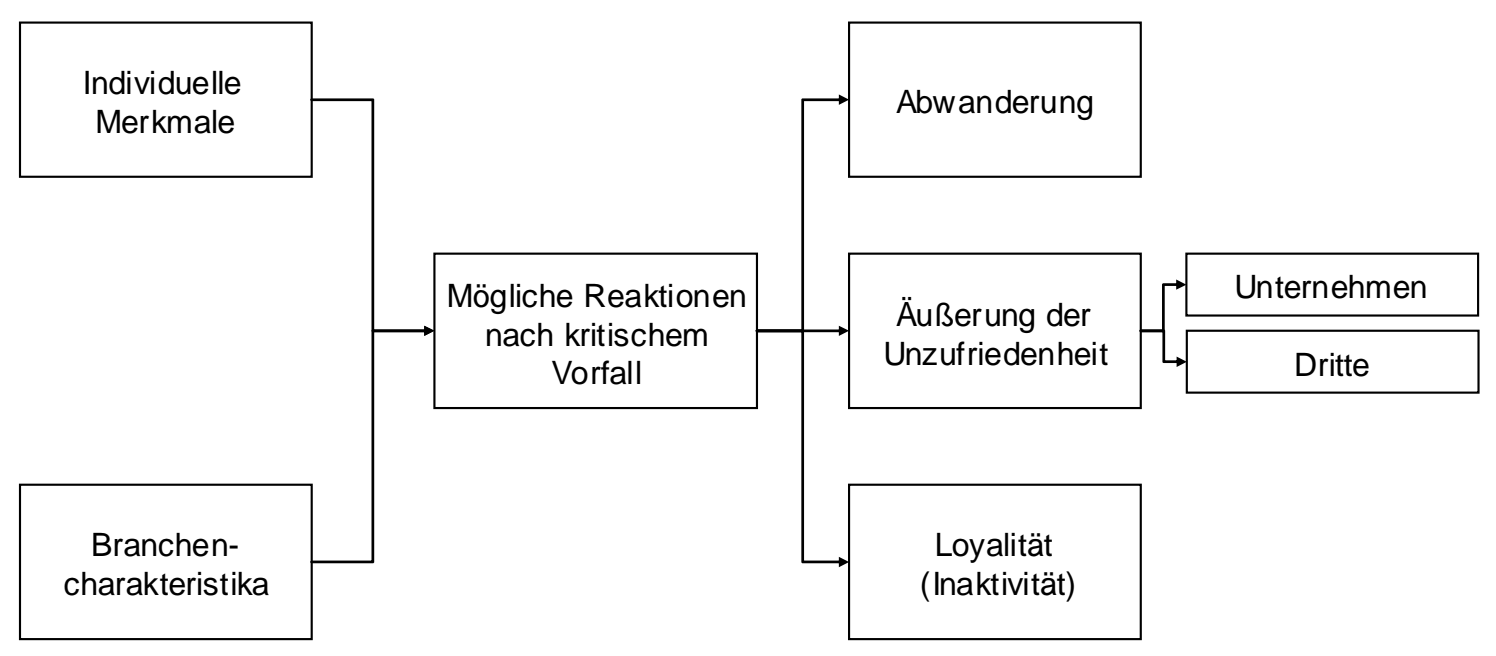

Auf der ersten Ebene wirken die Branchencharakteristika. Herrscht in der Branche eine hohe Wettbewerbsintensität, können die Kunden zwischen den konkurrierenden Unternehmen wählen und sind zumeist auch sehr gut hinsichtlich möglicher Alternativen informiert. Ist zudem die Branche von niedrigen Ein- und Austrittsgebühren (Wechselkosten) auf Seiten der Kunden gekennzeichnet (Singh 1990b), ist die Wechselwahrscheinlichkeit unzufriedener Kunden höher als die der Beschwerdeäußerung (Hirschman 1970). Der Unternehmens- und Branchenkontext des vorliegenden Beitrags lässt sich sehr gut in das dargestellte Szenario einordnen. Aufgrund der niedrigen Wechselkosten müsste auf Seiten der Kunden eine höhere Wechsel- als Beschwerdewahrscheinlichkeit vorliegen. Ferner erklärt das Modell die unterschiedlichen Kundenreaktionen nach einem negativen Vorfall auf Basis individueller Merkmale. Dabei kristallisieren sich zwei Erklärungsansätze hinsichtlich einer möglichen Beschwerdeäußerung heraus: Je niedriger die wahrgenommen „Kosten“ (zeitliche und finanzielle Aufwendungen) und je höher die Erfolgswahrscheinlichkeit (Einflussnahme und potenzielle Wiedergutmachung) sind, desto wahrscheinlicher ist die Beschwerdeäußerung gegenüber dem betroffenen Unternehmen (Hirschman 1970). Sofern der negative Vorfall zur Unzufriedenheit des Kunden führt, kann dieser laut Hirschman (1970) wie folgt reagieren: (1) Die erste Alternative besteht in der Beendigung der Beziehung zu dem Unternehmen und dem damit verbundenen Anbieterwechsel. (2) Die zweite Alternative kann sowohl die Beschwerdeäußerung gegenüber dem Unternehmen als auch negative Äußerungen gegenüber Dritten umfassen. (3) Letztlich kann der Kunde auch ohne seine Beschwerde zu artikulieren die Beziehung zum Unternehmen einfach fortführen.

Weitere Anhaltspunkte zur Bedeutung von Input-Outcome-Relationen, welche zentral für die Entscheidung zur und Beurteilung der Beschwerde sind, gibt die Equity-Theorie 
(Adams 1963). Demnach vergleichen Individuen stets den geleisteten Input mit dem erhaltenen Outcome und stellen diese einem internen bzw. externen Vergleichsmaßstab gegenüber. Entspricht das individuelle Verhältnis zwischen Input (resp. Investitionen) und Outcome dem Verhältnis des Anbieters (externer Vergleichsmaßstab), so wird die Beziehung als fair wahrgenommen und damit Aufrecht erhalten. Kunden vergleichen dabei auch regelmäßig diese Verhältnisse mit denen anderer Anbieter, um zu bestimmen, was als fair gilt. Für die Beschwerdeforschung sind die Erkenntnisse der Equity-Theorie von grundlegender Bedeutung (Maxham 2001; Voorhees et al. 2006). Im Falle eines negativen Vorfalls, der auf Seiten des Kunden Unzufriedenheit auslöst, entsteht ein unausgewogenes Input-OutcomeVerhältnis auf Seiten des Kunden im Vergleich zum Anbieter. Der Kunde leistet durch den Kauf des Produktes oder der Dienstleistung Input in die Beziehung und erwartet zumindest eine dem Input entsprechende Gegenleistung. Im Falle von Unzufriedenheit besteht ein unausgewogenes Verhältnis zwischen den Input-Outcome-Relationen. Die Austauschbeziehung wird als unfair empfunden, da die Relation von Input und Outcome des Kunden relativ schlechter als die des Anbieters wahrgenommen wird. Als Konsequenz nimmt die Bereitschaft des Kunden, die Geschäftsbeziehung zu verlassen, zu.

\section{Kundenbindung von Noncomplainern}

\subsection{Herleitung der Untersuchungshypothesen}

Die übergeordnete Forschungsfrage des vorliegenden Beitrags lautet: Welches Bindungsniveau weisen Noncomplainer nach einem negativen Vorfall gegenüber dem betreffenden Unternehmen im Vergleich zu anderen Kundengruppen auf? Vor diesem Hintergrund gilt es zu prüfen, ob sich die Noncomplainer in ihrer Bindung gegenüber dem Unternehmen und insbesondere in ihrem tatsächlichen Kaufverhalten nach einem negativen Vorfall von den anderen Kundengruppen (Complainer und Kunden ohne Vorfall) unterscheiden. Ist dies nicht der Fall, bedarf es auch keiner Stimulation dieser Kunden zur Äußerung ihrer Beschwerde, da ihr Kaufverhalten nach dem Vorfall nicht negativ von diesem Vorfall beeinflusst wird (obgleich mit negativer Mundpropaganda oder sonstigem opportunistischem Verhalten zu rechnen wäre).

Um differenzierte Aussagen zu den Einstellungen des Kunden gegenüber dem Unternehmen und dessen Verhalten treffen $\mathrm{zu}$ können, wird im Folgenden der 
Kundenbindungsdefinition von Oliver (1997) gefolgt. Demnach beschreibt die Kundenbindung eine tief empfundene Verpflichtung, ein bestimmtes Produkt oder eine Dienstleistung zukünftig wiederzukaufen oder weiterzufördern, wodurch ein wiederholter Kauf derselben Marke oder derselben Marken-Gruppe bewirkt wird, trotz situationsbedingter Einflüsse und Marketing-Bemühungen, die das Potenzial haben, einen Wechsel im Kaufverhalten zu bewirken.

Oliver (1997, 1999) unterscheidet zwischen einer kognitiven, affektiven, konativen sowie aktionalen Loyalität, wobei die aktionale Komponente in dieser Studie mittels des tatsächlichen Kaufverhaltens nach dem Vorfall operationalisiert wird. Im Einzelnen sind die Stufen wie folgt zu beschreiben: (1) Die kognitive Loyalität äußert sich in einem rationalen Gefühl der Vorziehenswürdigkeit des ausgewählten Objekts gegenüber den verfügbaren Alternativen. Diese Form der Kundenbindung basiert maßgeblich auf dem vom Kunden wahrgenommenen Nettonutzen. Die Loyalität ist das Ergebnis eines kognitiven Vergleichsprozesses, der auf einem reinen Kosten-Nutzenkalkül basiert (vgl. Evanschitzky/Wunderlich 2006; Harris/Goode 2004; Kalyanaram/Little 1994; Sivakumar/Raj 1997). (2) Die affektive Loyalität basiert auf der Zufriedenheit des Kunden. Sie resultiert damit nicht nur aus Kognitionen, sondern verstärkt aus affektiven Größen wie der Gesamtzufriedenheit nach dem Kauf (vgl. Oliver 1999, S. 35.). Die affektive Loyalität entsteht durch wiederholte Transaktionen, in denen der Kunde positive Erfahrungen mit einem Unternehmen gemacht hat. (3) Bei der konativen Loyalität handelt es sich um positive Verhaltensabsichten des Kunden, ein bestimmtes Produkt oder Dienstleistung wieder zu kaufen (oder zu empfehlen). Der Kunde beabsichtigt und ist motiviert, eine bestimmte Leistung erneut in Anspruch zu nehmen; ob diese Absicht aber tatsächlich im Verhalten mündet, bleibt auf dieser Stufe jedoch unklar. (4) Die Umsetzung der Verhaltensabsicht in die Realisierung des Treueverhaltens bezeichnet Oliver (1999) als aktionale Loyalität. Um dieses Treueverhalten entwickeln zu können, muss es dem Kunden gelingen, sämtliche Hindernisse $\mathrm{zu}$ überwinden, die der Realisierung der Handlung im Wege stehen (vgl. Evanschitzky/Wunderlich 2006). Diese vier Formen der Kundenbindung stehen in folgendem kausalen Zusammenhang - wie etablierte Einstellungstheorien darlegen [2]:

\section{Kognition $\rightarrow$ Affektion $\rightarrow$ Konation $\rightarrow$ Verhalten.}

Im Folgenden werden auf Basis der beiden theoretischen Ansätze Untersuchungshypothesen zur vergleichenden Analyse der Kundengruppen hergeleitet. Dabei 
wird die Gruppe der Noncomplainer den Kundengruppen der (zufriedenen und unzufriedenen) Complainer gegenübergestellt, wobei die Kunden ohne jeglichen Vorfall als Kontrollgruppe fungieren. Wir differenzieren zwischen den zufriedenen und unzufriedenen Complainern, da in der Literatur oftmals im Rahmen des sog. Service Recovery Paradoxon argumentiert wird, dass durch erfolgreiche Beschwerdebearbeitung das Bindungsniveau der Kunden über das ursprüngliche Niveau hinaus gesteigert werden kann (vgl. De Matos et al. 2007 sowie die dort zitierte Literatur). Zur Kontrolle dieses etwaigen Effekts bedarf es einer entsprechenden Differenzierung zwischen den beiden Typen von Complainern.

Die Gruppeneffekte werden in diesem Abschnitt sukzessive hergeleitet und in mehreren Hypothesen formuliert. Vor diesem Hintergrund wird zunächst die Kundengruppe der unzufriedenen Complainer mit den übrigen Gruppen verglichen. Grundsätzlich entsteht Unzufriedenheit, wenn die Ist-Leistung negativ von der erwarteten Soll-Leistung abweicht und somit eine negative Diskonfirmation erfolgt (Oliver 1997). Demnach sind die unzufriedenen Complainer bereits aufgrund des negativen Vorfalls unzufrieden. Die Ausführungen zu Hirschmans Exit-Voice-Loyalty-Theorie zeigen, dass Kunden verschiedene Reaktionsmöglichkeiten auf Unzufriedenheit besitzen. Eine Option besteht in der Beschwerdeartikulation gegenüber dem Anbieter, wodurch der Kunde seine Unzufriedenheit in Richtung des Anbieters kanalisiert und ihn damit zur Behebung des Fehlers zwingt. Erfolgt allerdings eine nur unzureichende Behebung des Fehlers, steigt das Unzufriedenheitsniveau des Kunden weiter an (Voorhees et al. 2006; Keaveney 1995). Dieser Anstieg ist mit dem nunmehr noch schlechteren Verhältnis von geleistetem Input und erhaltenem Outcome in Relation zu dem Verhältnis des Anbieters zu begründen, da der Kunde Aufwendungen mit der Artikulation der Beschwerde verbindet, ohne eine entsprechende Gegenleistung zu erhalten. Das zweimalige Versagen eines Anbieters wird in der Beschwerdeforschung als „Double Deviation“-Effekt bezeichnet (Maxham/Netemeyer 2002). Voorhees et al. (2006) zeigen, dass eine unzureichende Beschwerdebehandlung zu einem Absinken der Wiederkaufabsicht führt. Diese Ergebnisse werden auch von Keaveney (1995) untermauert, die feststellt, dass 17\% der Kunden aufgrund einer unbefriedigenden Beschwerdebehandlung den Anbieter wechseln. Vor diesem Hintergrund lässt sich folgende Hypothese ableiten:

$\mathrm{H}_{1}$ : Kunden, die sich aufgrund eines negativen Vorfalls beschweren und anschließend eine unbefriedigende Beschwerdebehandlung erfahren, weisen ein niedrigeres Kundenbindungsniveau auf als (a) zufriedene Complainer, (b) Noncomplainer und (c) Kunden ohne jeglichen Vorfall. 
Während es nahezu intuitiv ist, dass Kunden, die sowohl durch den Vorfall als auch durch die Bearbeitung der Beschwerde negative Erfahrungen gemacht haben, das niedrigste Niveau aufweisen, ist bislang nur wenig über die Kundenbindung von Noncomplainern bekannt [3]. Erste Ergebnisse zu diesem speziellen Thema der Beschwerdeforschung liefert eine Studie aus dem Jahre 1979 (vgl. TARP 1979). Die Ergebnisse der Untersuchung verdeutlichen, dass mehr als $60 \%$ der Befragten, die ihre Unzufriedenheit nicht gegenüber dem Anbieter artikulierten, einen Wiederkauf des Produktes oder der Dienstleistung nicht beabsichtigten (TARP 1986). Untermauert werden diese Erkenntnisse von jüngeren Forschungsergebnissen. So zeigen Voorhees et al. (2006), dass die Gruppe der Noncomplainer eine niedrigere Wiederkaufabsicht aufweist als Complainer, deren Beschwerde zur Zufriedenheit gelöst worden ist. Theoretisch können diese Erkenntnisse mit der Equity-Theorie erklärt werden. Noncomplainer äußern die Unzufriedenheit nicht gegenüber dem Anbieter und liefern somit auch keinen Input. Dieses Verhalten bedingt allerdings, dass Noncomplainer auch keinen Outcome in Form einer Wiedergutmachung erhalten. Folglich besteht bei dieser Kundengruppe, bedingt durch die unbefriedigende Transaktion, ein unausgewogenes Verhältnis zwischen Input und Outcome, womit auch die Relation von Input und Outcome im Vergleich zum Anbieter unausgewogen ist, weshalb die folgende Hypothese formuliert werden kann:

$\mathrm{H}_{2}$ : Noncomplainer weisen ein niedrigeres Kundenbindungsniveau auf als (a) zufriedene Complainer und (b) Kunden ohne Vorfall.

In der Literatur existieren widersprüchliche Ergebnisse hinsichtlich der Kundenbindung nach der Beschwerdebearbeitung. Einige Studien weisen bspw. die Existenz des Service Paradoxons nach, wohingegen andere Untersuchungen dieses widerlegen. Das Paradoxon besagt, dass Kunden ohne jeglichen Vorfall ein niedrigeres Kundenbindungsniveau aufweisen als zufriedene Complainer (De Matos et al. 2007). Vor dem Hintergrund der Equity-Theorie erscheint diese Erkenntnis allerdings eher kontraintuitiv (dennoch kontrollieren wir diesen Effekt durch Differenzierung zwischen zufriedenen und unzufriedenen Complainern). So erfordert die Beschwerdeartikulation eine erhöhte Input-Leistung des unzufriedenen Kunden. Wenn die Beschwerde zur Zufriedenheit bearbeitet und der entstandene Schaden kompensiert worden ist, wird lediglich ein ausgeglichenes Verhältnis zwischen Input und Outcome 
wiederhergestellt [4]. Analog zu diesen Überlegungen konnte Bruhn (1998) empirisch nachweisen, dass Kunden ohne jeglichen Vorfall durchgängig das höchste Kundenbindungsniveau aufwiesen. Auf Basis dieser Überlegungen und der empirischen Ergebnisse erscheint bei einer reinen Kompensation des entstandenen Schadens eine Steigerung des Kundenbindungsniveaus nicht plausibel, weshalb zu vermuten ist:

$\mathrm{H}_{3}$ : Kunden ohne Vorfall weisen das höchste Kundenbindungsniveau auf.

Die Hypothesen $\mathrm{H}_{1}-\mathrm{H}_{3}$ lassen sich in Form der folgenden Ungleichung zusammenfassen, welche auf das Niveau der Kundenbindung in den vier Kundengruppen abstellt:

Kunden ohne Vorfall $\left(\mathrm{H}_{3}\right)>$ zufriedene Complainer $>$ Noncomplainer $\left(\mathrm{H}_{2}\right)>$ unzufriedene Complainer $\left(\mathrm{H}_{1}\right)$

\subsection{Datenerhebung und Stichprobencharakteristika}

Zur Überprüfung der Untersuchungshypothesen wurde ein Unternehmen der Systemgastronomie ausgesucht, da dieses eine hohe Transaktionsquote (Kauffrequenz im 14tägigen Rhythmus) der Kunden garantiert. Diese ist für die vorliegende Untersuchung von großer Bedeutung, da eine Rekapitulation des negativen Vorfalls durch den Kunden lediglich innerhalb der letzten sechs Monate valide ist [5]. Zudem sollte der Unternehmenskontext durch einen hohen Wettbewerb und niedrige Wechselkosten gekennzeichnet sein, damit garantiert werden kann, dass potenziell unerwünschte Einflussfaktoren auf das Beschwerdeverhalten, wie bspw. durch eine vertragliche Bindung hervorgerufen, kontrolliert werden. Das hier betrachtete Unternehmen gehört den Delivery-Services an und teilt sich mit mehr als 2.700 weiteren Anbietern dieses Marktsegment. Insgesamt wurden an 126 Standorten jeweils 75 zufällig ausgewählte Kunden dieses Unternehmens telefonisch befragt [6]. Uns wurde die vollständige Kundendatenbank des Unternehmens zur Verfügung gestellt, die alle Kunden (sowohl aktive als auch inaktive) umfasste. Damit konnten wir einem möglichem Selection Bias vorbeugen, da bspw. Kunden, die aufgrund von Unzufriedenheit inaktiv sind, in unserer Zufallsauswahl enthalten sind. Die Teilnahmebereitschaft der Probanden konnte als sehr positiv beurteilt werden: Es erklärten sich ca. $30 \%$ der Angerufenen zu einer Teilnahme an der Befragung bereit. Die 70\% Abbrecher beendeten die 
Umfrage vor Nennung des Themas und des Unternehmens. Ferner zeigen sie keine signifikanten Unterschiede im Kaufverhalten im Vergleich zu den teilnehmenden Kunden und zu der Grundgesamtheit sämtlicher Kunden. Dementsprechend ist eine Selbstselektion unwahrscheinlich. Insgesamt konnten damit 9.461 auswertbare Interviews erhoben werden. Eine zusammenfassende Beschreibung der Stichprobe zeigt Tabelle 1.

Tab. 1: Demografischer Vergleich der Kundengruppen (Gesamtstichprobe)

\begin{tabular}{lccc}
\hline Beschreibende Merkmale & $\begin{array}{c}\text { Kunden ohne } \\
\text { Vorfall (n= 9.073) }\end{array}$ & $\begin{array}{c}\text { Noncomplainer } \\
(\mathbf{n}=\mathbf{1 5 5})\end{array}$ & $\begin{array}{c}\text { Complainer } \\
(\mathbf{n}=\mathbf{2 3 3})\end{array}$ \\
\hline$\emptyset$ Alter & 32,4 & 29,6 & 30,7 \\
Anteil Frauen (\%) & 58,4 & 61,0 & 53,6 \\
Anteil Männer (\%) & 41,6 & 39,0 & 46,4 \\
Einkommensklasse (\%) & & & \\
$\quad 0-1.000 €$ & 27,6 & 25,8 & 30,0 \\
$1.000 €-2.000 €$ & 35,0 & 38,7 & 32,0 \\
$2.000 €-3.000 €$ & 20,7 & 17,2 & 17,3 \\
mehr als 3.000€ & 16,7 & 18,3 & 20,7 \\
Familienstand (\%) & & & \\
$\quad$ Ledig & 52,4 & 47,4 & 55,2 \\
$\quad$ verheiratet/zusammenlebend & 47,6 & 52,6 & 44,8 \\
\hline
\end{tabular}

Zur Differenzierung zwischen den verschiedenen Beschwerdetypen wurden die Probanden nach einem negativen Vorfall innerhalb der letzten sechs Monate befragt. Dabei wurden nur Vorfälle erhoben, die beim Kunden auch Unzufriedenheit ausgelöst haben. Weiterhin wurden die Probanden gebeten, den Zeitpunkt des Vorfalls anzugeben, da sowohl das Kaufverhalten vor als auch nach dem Vorfall in die Untersuchung einbezogen wird, weshalb die zeitliche Einordnung des Vorfalls von hoher Bedeutung ist. Die Transaktionsdaten konnten somit kundenindividuell in die Zeiträume vor bzw. nach dem Vorfall unterteilt werden.

\subsection{Messmodell}

Die Messung der latenten Konstrukte erfolgte unter Heranziehung früherer Studien der Kundenbindungs- und Beschwerdeliteratur. Da Oliver (1999) zwischen einer kognitiven, affektiven, konativen und aktionalen Komponente der Kundenbindung differenziert, wurde die kognitive Loyalität in Anlehnung an Dodds und Monroe (1991) sowie an Sirdeshmukh, Singh und Sabol (2002) gemessen, da diese Items das Kosten-Nutzenkalkül wiedergeben (vgl. Evanschitzky/Wunderlich 2006). Eine ähnliche Operationalisierung wird auch von Harris und Goode (2004) vorgenommen. Die Messung der affektiven Loyalität erfolgte wie von Oliver 
(1997) empfohlen mittels der „Gesamtzufriedenheit“ der Kunden; die Items wurden in Anlehnung an Evanschitzky und Wunderlich (2006) bzw. Bettencourt (1997) adaptiert. Die konative Loyalität (Verhaltensabsicht) basiert auf einer Skala von Zeithaml, Berry und Parasuraman (1996) und umfasst die Wiederkauf- und die Weiterempfehlungsabsicht der Kunden. Sowohl bei den Complainern als auch den Noncomplainern wurden die Indikatoren der konativen Loyalität kontextspezifisch angepasst. Diese Skala stammt im Kontext der Beschwerdeforschung von Homburg und Fürst (2005) und umfasst sowohl Indikatoren zur konativen als auch zur aktionalen Loyalität (tatsächliches Verhalten) ergänzt um die zeitliche Angabe „nach der Beschwerde.“ Von diesem Ansatz wird im Folgenden mit Blick auf die im Kontext der Common-Method-Bias Diskussion (Podsakoff et al. 2003) vorgebrachten Argumente Abstand genommen, da das Verhalten gesondert erfasst wurde. Somit wird die konative Loyalität von Complainern und Noncomplainern mit zwei Indikatoren gemessen, die sich lediglich auf die Verhaltensabsicht nach der Beschwerde bzw. nach dem Vorfall beziehen. Die aktionale Loyalität wurde mittels der kundenindividuellen Umsätze nach der Beschwerde gemessen. Des Weiteren erfolgte die Differenzierung zwischen den zufriedenen und den unzufriedenen Complainern anhand der Beschwerdezufriedenheitsskala von Homburg und Fürst (2005). Sämtliche Skalen wurden auf Basis einer 5-Punkte Likert Skala mit den Polen $1=$,trifft voll zu“ und 5 = „trifft gar nicht $\mathrm{zu}^{\text {“ }}$ gemessen (vgl. zu den Items Tab. 2).

\subsection{Ergebnisse}

Die Reliabilität des Messmodells wurde durch eine konfirmatorische Faktorenanalyse und die Berechnung der Cronbach's Alpha Koeffizienten überprüft. Tabelle 2 fasst die Gütekriterien des Messmodells zusammen und umfasst sowohl die Faktorreliabilitäten als auch die Werte der einzelnen Cronbach's Alphas. Sämtliche Cronbach's Alpha-Werte und Faktorreliabilitäten sind größer als die in der Literatur geforderten Schwellenwerte. Auch die durchschnittlich erklärte Varianz nimmt durchgehend zufrieden stellende Werte an. Hinsichtlich der globalen Gütemaße liefert das Messmodell ebenfalls eine akzeptable Anpassung an die empirische Datenstruktur (CFI $=0,939$; $\mathrm{TLI}=0,912$; $\mathrm{RMSEA}=0,092$; $\mathrm{SRMR}=0,061)$ [7]. Im Rahmen der Faktoranalyse musste jedoch das Item „Cross-BuyingIntention“ auf Ebene der konativen Loyalität eliminiert werden. Es wies zwar eine Faktorladung über 0,5 auf, jedoch trug es zu einer wesentlichen Verschlechterung der Detailund Globalkriterien bei. 
Tab. 2: Indikatoren und Gütekriterien des Messmodells

\begin{tabular}{|c|c|c|c|c|}
\hline Konstrukt & Indikatoren & $\begin{array}{c}\text { Cronbach's } \\
\text { Alpha }\end{array}$ & $\begin{array}{c}\text { Faktor- } \\
\text { reliabilität }\end{array}$ & $\begin{array}{c}\text { Durch. erkl. } \\
\text { Varianz }\end{array}$ \\
\hline Kognitive & Das Preisniveau des Anbieters ist gut. & & & \\
\hline Loyalität & $\begin{array}{l}\text { Das Preis-Leistungs-Verhältnis des Anbieters ist } \\
\text { gut. } \\
\text { Der Service des Anbieters ist das Geld wert. }\end{array}$ & 0,84 & 0,85 & 0,65 \\
\hline Affektive & Gesamtzufriedenheit mit dem Anbieter & & & \\
\hline Loyalität & $\begin{array}{l}\text { Mein Anbieter ist besser als vergleichbare } \\
\text { Anbieter vor Ort. } \\
\text { Ich habe bisher gute Erfahrungen mit dem } \\
\text { Anbieter gemacht. }\end{array}$ & 0,87 & 0,88 & 0,71 \\
\hline $\begin{array}{l}\text { Konative } \\
\text { Loyalität }\end{array}$ & $\begin{array}{l}\text { Es ist sehr wahrscheinlich, dass ich nach dem } \\
\text { Vorfall wieder bei dem Anbieter kaufe. } \\
\text { Zukünftig werde ich dem Anbieter gegenüber treu } \\
\text { bleiben. }\end{array}$ & 0,90 & 0,90 & 0,82 \\
\hline $\begin{array}{l}\text { Beschwerde- } \\
\text { zufriedenheit }\end{array}$ & $\begin{array}{l}\text { Ich war mit der Beschwerdebearbeitung sehr } \\
\text { zufrieden. } \\
\text { Ich habe bei der Beschwerde positive } \\
\text { Erfahrungen mit dem Anbieter gemacht. } \\
\text { Der Anbieter hat meine Beschwerde zur äußersten } \\
\text { Zufriedenheit gelöst. }\end{array}$ & 0,96 & 0,96 & 0,89 \\
\hline
\end{tabular}

Als Methode zur Beantwortung der in der Einleitung aufgeworfenen Forschungsfragen findet im Folgenden die Varianzanalyse Anwendung. Die Varianzanalyse stellt insbesondere in internationalen Forschungsbeiträgen zum Konsumentenverhalten eine der am häufigsten angewendeten Methoden dar [8]. Daher gilt es, neben der Überprüfung des Messmodells die Anforderungen der Varianzanalyse an die Daten zu überprüfen. Dazu wurde die Stichprobe zunächst auf potenzielle Ausreißer untersucht, wobei in der vorliegenden Untersuchung lediglich zwei Ausreißer eliminiert wurden, da diese im Vergleich zu den anderen Probanden eine fast dreifach höhere Standardabweichung des Umsatzes aufwiesen. Die Gruppenzuordnung erfolgte ex ante durch die Auskunft der Probanden, wodurch eine Gruppengröße von mehr als 20 Probanden je Kundengruppe ebenfalls erfüllt wurde. Zur Differenzierung der Kundengruppen wurde gefragt, ob die Kunden in den letzten sechs Monaten einen negativen Vorfall in Verbindung mit ihrem Kauf hatten und ob sie sich beschwert bzw. nicht beschwert haben.

Die Varianzhomogenität zwischen den zu untersuchenden Gruppen und die geforderte multivariate Normalverteilung der Daten wurde nicht erfüllt, was allerdings durch die Gleichheit der jeweiligen Gruppengröße und einer randomisierten Gleichbesetzung der Zellen behoben wurde (Eschweiler 2006; Glaser 1978). Dementsprechend qualifiziert sich der Datensatz auch für die weitere Überprüfung der Hypothesen mittels Varianzanalyse. 
Zur Überprüfung der Hypothesen wird in einem paarweisen Vergleich die Gruppe der Noncomplainer mit den Gruppen der zufriedenen und unzufriedenen Complainern sowie der Kontrollgruppe (Kunden ohne Vorfall) verglichen werden. Die Einteilung in zufriedene und unzufriedene Complainer erfolgte anhand eines einfachen Mediansplits mittels der Variable Beschwerdezufriedenheit. Da 70 Noncomplainern eine Umsatzgröße zugeordnet werden konnte, musste zur Behebung der nicht vorhandenen Varianzhomogenität jede in der Untersuchung integrierte Kundengruppe auf 70 Probanden reduziert werden. Insgesamt konnten so 70 zufriedene und 70 unzufriedene Complainer extrahiert werden und in die Untersuchung einfließen [9]. Die Ergebnisse der Varianzanalyse zeigen, dass die ersten drei Formen der Kundenbindung (affektiv, kognitiv und konativ) signifikante Unterschiede zwischen den vier Kundengruppen aufweisen, wohingegen die beobachtete Größe der aktionalen Kundenbindung (Umsatz nach dem Vorfall) nicht signifikant ist (vgl. Tab. 3).

Tab 3: Ergebnisse der Varianzanalyse (1)

\begin{tabular}{lccccc}
\hline Variable & $\begin{array}{c}\text { Unzufriedene } \\
\text { Complainer }\end{array}$ & $\begin{array}{c}\text { Zufriedene } \\
\text { Complainer }\end{array}$ & $\begin{array}{c}\text { Non- } \\
\text { complainer }\end{array}$ & $\begin{array}{c}\text { Kontrollgrup } \\
\text { pe }\end{array}$ & F-Wert \\
\hline Kognitive Loyalität & 2,58 & $2,29 *$ & 2,79 & $2,00 * * *$ & 14,99 \\
Affektive Loyalität & 3,25 & $2,56^{* * *}$ & 2,98 & $1,86^{* * *}$ & 32,09 \\
$\begin{array}{l}\text { Konative Loyalität } \\
\text { (gesamt) }\end{array}$ & 2,56 & $1,76^{* * *}$ & 2,29 & $1,40^{* * *}$ & 16,48 \\
$\begin{array}{l}\text { Konative Loyalität } \\
\text { (nach dem Vorfall) }\end{array}$ & 2,75 & $1,82^{* * *}$ & 2,67 & - & 12,59 \\
$\begin{array}{l}\text { Aktionale Loyalität } \\
\text { (Umsatz) }\end{array}$ & 64,73 & 88,83 & 61,16 & - & 2,04 \\
\hline
\end{tabular}

\begin{tabular}{l} 
Anmerkungen: \\
$* 0,9-$ Niveau signifikant \\
$* * 0,95-$ Niveau signifikant \\
$* * * 0,99-$ Niveau signifikant \\
\hline
\end{tabular}

Hypothese $\mathrm{H}_{1}$ nimmt an, dass die unzufriedenen Complainer im Vergleich zu den anderen Kundengruppen das niedrigste Kundenbindungsniveau aufweisen. Die Ergebnisse der paarweisen Vergleiche zeigen im Einzelnen, dass Hypothese $\mathrm{H}_{1}$ teilweise bestätigt werden kann. Unzufriedene Complainer weisen - entgegen unserer Vermutung - hinsichtlich der kognitiven, affektiven und konativen (gesamt) Kundenbindung ein ähnlich niedriges Kundenbindungsniveau auf wie die Noncomplainer. Dagegen weisen die unzufriedene Complainer - wie vermutet - signifikant niedrigere Werte gegenüber den zufriedenen Complainern und der Kontrollgruppe auf. Auch bei der konativen Kundenbindung (nach dem Vorfall) haben die unzufriedenen Complainer erneut ein den Noncomplainern ähnlich niedriges Kundenbindungsniveau und gleichzeitig signifikant schlechtere Werte als die 
zufriedenen Complainer. Bei einem Vergleich der Noncomplainer mit den unzufriedenen Complainern ist demnach festzustellen, dass kein signifikanter Unterschied zwischen den Gruppen besteht, weshalb die Hypothese $\mathrm{H}_{1 \mathrm{~b}}$ abgelehnt werden muss. Hinsichtlich der aktionalen Kundenbindung ist anzumerken, dass in der Stichprobe zwar eine tendenzielle Veränderung des Kaufverhaltens zu beobachten ist. Aufgrund des nicht signifikanten F-Tests $(\mathrm{F}=2,04)$ kann aber nicht auf entsprechende Unterschiede in der Grundgesamtheit sämtlicher Kunden geschlossen werden.

Hypothese $\mathrm{H}_{2}$ nimmt an, dass Noncomplainer ein niedrigeres Kundenbindungsniveau aufweisen als zufriedene Complainer und Kunden ohne Vorfall. Dazu wird auf Basis eines paarweisen Gruppenvergleichs die Gruppe der Noncomplainer als Referenzgruppe zugrunde gelegt (vgl. Tab 4). Bei Betrachtung der Ergebnisse ist festzustellen, dass die Noncomplainer auf sämtlichen Stufen der Kundenbindung signifikant schlechtere Werte aufweisen, weshalb Hypothese $\mathrm{H}_{2}$ durch die empirischen Daten bestätigt wird.

Tab 4: Ergebnisse der Varianzanalyse (2)

\begin{tabular}{lcccc}
\hline Variable & Noncomplainer & $\begin{array}{c}\text { Zufriedene } \\
\text { Complainer }\end{array}$ & Kontrollgruppe & F-Wert \\
\hline Kognitive Loyalität & 2,79 & $2,29 * * *$ & $2,00^{* * *}$ & 19,63 \\
$\begin{array}{l}\text { Affektive Loyalität } \\
\text { Konative Loyalität } \\
\text { (gesamt) }\end{array}$ & 2,98 & $2,56^{* *}$ & $1,86^{* * *}$ & 29,80 \\
$\begin{array}{l}\text { Konative Loyalität } \\
\text { (nach dem Vorfall) }\end{array}$ & 2,29 & $1,76^{* *}$ & $1,40^{* * *}$ & 13,88 \\
$\begin{array}{l}\text { Aktionale Loyalität } \\
\text { (Umsatz) }\end{array}$ & 2,67 & $1,82^{* * *}$ & - & 18,07 \\
\hline Ammerkungen: & 61,16 & $88,83^{*}$ & - & 3,31 \\
\hline
\end{tabular}

Hypothese $\mathrm{H}_{3}$ nimmt an, dass Kunden ohne Vorfall das höchste Kundenbindungsniveau aufweisen. Die Tabellen 3 und 4 deuten darauf hin, dass neben den signifikanten Unterschieden zwischen den unzufriedenen Complainern und den Kunden ohne Vorfall (Tabelle 3) sowie den Noncomplainern und den Kunden ohne Vorfall (Tabelle 4) auch Unterschiede im Mittelwert der zufriedenen Complainer und der Kunden ohne Vorfall zu vermuten sind. Eine Prüfung dieser Unterschiede zeigt, dass auf der Stufe der kognitiven Kundenbindung signifikante Mittelwertdifferenzen in Höhe von $-0,27(p<0,05)$ vorliegen. Die größte Differenz ist auf der Stufe der affektiven Loyalität in Höhe von $-0,70(p<0,01) z u$ finden. Auf der konativen Stufe weisen zufriedene Complainer einen Mittelwert von 1,76 auf, 
die Kontrollgruppe dagegen einen Mittelwert von 1,40 und auch diese Differenz ist signifikant $(\mathrm{p}<0,05)$. Damit ist die Hypothese $\mathrm{H}_{3}$ nicht $\mathrm{zu}$ verwerfen, wohingegen das Service-Recovery-Paradoxon abzulehnen ist, da Kunden ohne Vorfall bessere Werte aufweisen als Kunden die eine zufrieden stellende Beschwerdebearbeitung erfahren haben $[10]$.

Fasst man die Ergebnisse zusammen, so zeigen diese, dass Unternehmen explizit zwischen der Gruppe der Complainer sowie der Gruppe der Noncomplainer unterscheiden sollten. Insbesondere neigen die Noncomplainer dazu, ein niedrigeres Kundenbindungsniveau aufzuweisen als Kunden, die eine zufrieden stellende Wiedergutmachung nach einem negativen Vorfall erfahren haben und Kunden, die keinen negativen Vorfall erleben mussten. Zusätzlich muss betont werden, dass Noncomplainer ein ähnlich niedriges Kundenbindungsniveau aufweisen wie Kunden, die neben dem negativen Vorfall zusätzlich noch eine unbefriedigende Beschwerdebehandlung erfahren haben. Dementsprechend ist bei Betrachtung der Umsätze der Noncomplainer vor dem Vorfall im Vergleich zu den Umsätzen nach dem Vorfall festzustellen, dass die Noncomplainer ihre Umsätze bei diesem Anbieter signifikant reduzieren $(\mathrm{t}=4,37 ; \mathrm{p}<0,01)$. Allerdings ist $\mathrm{zu}$ beachten, dass es sich beim Umsatz um eine absolute Größe handelt, die auch von der Beziehungslänge abhängt. Daher betrachten wir nochmals die standardisierte Größe der Zeitspanne zwischen zwei Bestellvorgängen. Vor dem Vorfall tätigten Noncomplainer alle 45,5 Tage einen Einkauf, nach dem Vorfall reduzierte diese Gruppe den Zeitraum auf 61,7 Tage $(\mathrm{t}=-1,916, \mathrm{p}<0,1)$. Das zeigt, dass die Reduktion des Umsatzes nicht nur mit den unterschiedlichen Betrachtungszeiträumen einhergeht. Gleichzeitig können durch Stimulierung der Noncomplainer zur Kundenbeschwerde sowie nach erfolgreicher Bearbeitung der Beschwerde die Umsätze wieder deutlich gesteigert werden (88,83 Euro im Vergleich zu 61,16 Euro in dem vorliegenden Betrachtungszeitraum). Somit besitzt die Beschwerdestimulierung das Potenzial, einen erheblichen Beitrag zum Unternehmenserfolg zu leisten - nicht zuletzt aufgrund der Größe dieser Kundengruppe von fast 50\%.

\section{Implikationen für Management und Forschung}

\subsection{Managementimplikationen}

Dieser Beitrag verfolgte das Ziel, das Bindungsniveau von Noncomplainern zu betrachten und mit anderen Kundengruppen zu vergleichen, um Rückschlüsse für die 
Beschwerdestimulierung zu ziehen. Im Ergebnis konnte festgestellt werden, dass die Noncomplainer erstens ihre Umsätze nach dem Vorfall signifikant reduzieren und zweitens durch Stimulierung der Kunden zur Beschwerde sowie nach erfolgreicher Beschwerdebearbeitung die Umsätze wieder signifikant steigen (vgl. Gruppe der zufriedenen Complainer). Noncomplainer weisen ein ähnlich niedriges Kundenbindungsniveau auf wie Kunden, die einen negativen Vorfall erlebt haben und zudem eine unbefriedigende Beschwerdebearbeitung. Dementsprechend sollten Noncomplainer zur Beschwerde motiviert werden, jedoch nur, sofern eine erfolgreiche Beschwerdebearbeitung erfolgt. Durch eine unzureichende Beschwerdebearbeitung sinkt das Kundenbindungsniveau nicht weiter, dennoch sind mit der Regulierung Aufwendungen verbunden. Aus Perspektive der EquityTheorie stellt die Beschwerdestimulierung allerdings nur die zweitbeste Alternative dar. Die erstbeste Reaktion ist die unternehmensseitige, proaktive Wiedergutmachung eines negativen Vorfalls (vgl. Voorhees et al. 2006), da diese mit keinerlei zusätzlichen Aufwendungen seitens der Kunden verbunden ist. Notwendige Voraussetzung einer solchen Maßnahme ist wiederum die Identifikation von negativen Vorfällen seitens des Unternehmens und damit auch von potenziellen Noncomplainern. Beispielsweise sind die Mitarbeiter im Kundenkontakt hinsichtlich des proaktiven Umgangs mit Fehlern (ob unternehmensseitig oder kundenseitig verursacht) zu schulen und entsprechende Routinen zum Umgang zu implementieren. Falls einem Unternehmen die Verfolgung dieser proaktiven Strategie nicht gelingt, sollte der zweitbeste Weg, die Stimulierung der Noncomplainer zur Beschwerde, ausgebaut werden.

Potenzielle Maßnahmen könnten bspw. die Erleichterung der Beschwerdeäußerung durch die Schaffung von einfach zu erreichenden Beschwerdekanälen sein (vgl. Homburg/Fürst 2003; Stauss/Seidel 2007). Des Weiteren kann die Unternehmung ihre positive Einstellung gegenüber Beschwerden im Sinne von Verbesserungsvorschlägen kommunizieren. Weiterhin könnten Unternehmen auch aktiv auf ihre Kunden zugehen, bspw. durch die Evaluation der Kundenzufriedenheit nach dem Kauf von Dienstleistungen oder Produkten mit hohem Fehlerpotenzial. Eine weitere Möglichkeit ist die systematische Kontaktierung von Kunden nach einer bestimmten Zeitspanne ohne Kauf. Dieses wird bereits bei einigen Versandunternehmen praktiziert, indem Kunden, welche nach einem gewissen Zeitraum nicht wieder bestellt haben, kontaktiert und nach ihrer Zufriedenheit resp. einem negativen Vorfall befragt werden. Diese Form des aktiven Kundenmanagement dürfte angesichts unserer Ergebnisse auch für andere Unternehmungen und Branchen betriebswirtschaftlich sinnvoll sein. 


\subsection{Zukünftiger Forschungsbedarf und Einschränkungen}

Wie sämtliche empirische Studien unterliegt auch die vorliegende Untersuchung einigen Einschränkungen. So wurde festgestellt, dass Noncomplainer ein signifikant niedrigeres Bindungsniveau aufweisen als bspw. zufriedene Complainer und Kunden ohne jeglichen Vorfall. Hier stellt sich die Frage, ob Noncomplainer nicht bereits vor dem Vorfall ein niedrigeres Niveau aufwiesen und sie daher nicht an einer Beschwerde - damit verbunden an einer Wiedergutmachung seitens des Unternehmens - interessiert sind. Noncomplainer präferieren in diesem Falle die Wechseloption. Bereits Diller (1996, S. 81 ff.) konnte konzeptionell zeigen, dass die Beschwerde im Falle von Unzufriedenheit ein Aspekt der Loyalität sein kann. Zukünftige Forschungsarbeiten sollten an dieser Stelle ansetzen. So haben bisherige Arbeiten zur Identifikation von Noncomplainern gezeigt, dass die gewonnenen „Profile“ dieser Kunden, bspw. auf Basis von Persönlichkeitsmerkmalen oder Demographika, häufig einer empirischen Validierung nicht standhielten. Gemeinsames Merkmal dieser Studien ist jedoch, dass das Bindungsniveau vor dem negativen Vorfall keine Beachtung fand. Vor diesem Hintergrund ist es umso entscheidender, dass die Loyalität vor dem Vorfall als Kontrollvariable integriert wird. Dementsprechend sollten zukünftig Longitudinalstudien durchgeführt werden, die Einstellungs- mit Befragungsdaten kombinieren und neben soziodemografischen Merkmalen insbesondere das Bindungsniveau der Kunden vor dem negativen Vorfall berücksichtigen. Hinsichtlich des Kaufverhaltens nach dem Vorfall ist in der vorliegenden Studie ferner der Beobachtungszeitraum nach dem Vorfall als weitere Limitation anzuführen. Auch wenn das betrachtete Unternehmen durch eine hohe Transaktionsfrequenz auf Seiten der Kunden gekennzeichnet ist, könnte der durchschnittliche Beobachtungszeitraum von sechs Monaten nach dem Vorfall ausgeweitet werden.

Ferner basiert die Studie lediglich auf der Betrachtung nur eines Unternehmens. Diese Fokussierung begründen wir damit, dass die Komplexität der Datenerhebung eine branchenund unternehmensübergreifende Analyse verhinderte. So ist die Zuordnung von Befragungsund Beobachtungsdaten, sowohl vor als auch nach einem negativen Vorfall, mit einer hohen Komplexität behaftet, so dass im Rahmen des vorliegenden Beitrags lediglich ein Unternehmen betrachtet werden konnte. Diese Fokussierung geht leider mit Einschränkungen der externen Validität einher. Weitere Forschungsarbeiten sollten dennoch branchenübergreifend durchgeführt werden, um bspw. Beschwerdebarrieren sowie damit verbundene Maßnahmen der Beschwerdestimulierung branchenspezifisch (B2B versus B2C) 
und auch in Abhängigkeit der Produkte und Dienstleistungen des Unternehmens (Involvement des Kunden, Wert des Objekts, verbundenes Risiko) zu identifizieren. Als letzte Limitation muss angeführt werden, dass im vorliegenden Beitrag der Annahme nachgegangen wird, dass Beschwerden grundsätzlich positiv zu betrachten sind. Allerdings ist eine zufrieden stellende Beschwerdebearbeitung mit Kosten verbunden. Vor diesem Hintergrund kann es durchaus im Sinne des Unternehmens sein, dass unrentable Kunden nicht zur Beschwerde motiviert werden und stattdessen den Anbieter wechseln. Aus diesen Einschränkungen heraus resultiert zugleich weiterer Forschungsbedarf für das Kundemanagement, explizit die Kosten der Beschwerderegulierung in zukünftige Untersuchungsdesigns zu integrieren. 


\section{Anmerkungen:}

[1] Wir legen bei diesem Beispiel die Annahme zugrunde, dass jeder Kunde nur ein Mal seine Beschwerde gegenüber dem Unternehmen artikuliert.

[2] An dieser Stelle ist bspw. die „Theory of Reasoned Action“ zu nennen, vgl. dazu insbesondere Fishbein/Ajzen (1975).

[3] Es existiert keine Studie, die das Konzept der Kundenbindung mit sämtlichen Facetten im Rahmen der Beschwerdeforschung untersucht. Bislang existieren wenige Studien, die die Auswirkungen des Beschwerde- und Noncomplainerverhaltens auf einzelne Facetten der Kundenbindung, wie bspw. der Wiederkaufabsicht oder der Gesamtzufriedenheit, analysieren (Voorhees et al. 2006).

[4] Diese Aussage trifft allerdings nur unter der Annahme zu, dass keine Überkompensation des Vorfalls stattgefunden hat. Es ist denkbar, dass eine Überkompensation vor dem Hintergrund des C/D-Paradigmas zu einem höheren Zufriedenheits- und damit auch zu einem höheren Loyalitätsniveau führen könnte als ohne jeglichen Vorfall. Diese Annahme wurde zwar durch die Studie von Garret (1999) widerlegt, eine Validierung der Ergebnisse steht jedoch noch aus. Eine reine Kompensation des Fehlers und die zufrieden stellende Beschwerdebearbeitung, wie es in der vorliegenden Untersuchung gehandhabt wurde, führen somit nicht zu einem erhöhten Kundenbindungsniveau (Zeithaml et al. 1996).

[5] Vgl. zur Problematik potenzieller Verzerrungen des Antwortverhaltens hervorgerufen durch Erinnerungslücken und Rationalisierungstendenzen (Smith/Bolton 1998).

[6] Insgesamt konnten 9.461 Kundenbefragungen durchgeführt werden. Aufgrund von Pretests ergibt sich in Summe ein höheres $n$ als rechnerisch erwartet (9.450).

[7] Aufgrund der nicht vorhandenen Varianzhomogenität haben wir versucht, die Verletzung über eine Gleichbesetzung der Zellen zu heilen (vgl. Erläuterung im Text). Die Gütekriterien wurden auf Basis der kleineren Stichprobe $(\mathrm{n}=280)$ berechnet.

Die Korrelationen zwischen den einzelnen Konstrukten weisen nahezu durchgängig akzeptable Werte auf. Lediglich die Korrelation zwischen der affektiven und konativen Loyalität erreicht einen Wert nahe 0,79. Dies schränkt die Diskriminanzvalidität zwischen den beiden Faktoren ein; stellt allerdings in der vorliegenden Untersuchung kein schwerwiegendes Problem dar, da lediglich eine vergleichende Analyse der unterschiedlichen Kundenbindungsstufen durchgeführt wird und die Konstrukte jeweils zu Faktormittelwerten zusammengefasst werden. Bei 
Anwendung einer Kovarianz-Strukturanalyse müsste jedoch versucht werden, die Diskriminierung zwischen den beiden Konstrukten zu gewährleisten.

[8] So waren bspw. in den letzten zwei Jahrzehnten mehr als 60\% der Studien im Journal of Consumer Research varianzanalytisch ausgerichtet (vgl. insbes. Eschweiler/Evanschitzky/Woisetschläger 2007, S. 3).

[9] Nachfolgend sind die wesentlichen demografische und kundenbezogene Merkmale der reduzierten Stichprobe $(\mathrm{n}=280)$ zusammengefasst:

\begin{tabular}{lc}
\hline Demografische Merkmale & \\
\hline$\varnothing$ Alter & $30,76(\mathrm{SD}=10,18)$ \\
Anteil Frauen (\%) & 57,1 \\
Anteil Männer (\%) & 42,9 \\
Einkommensklasse (\%) & \\
$0-1.000 €$ & 30,6 \\
$1.000 €-2.000 €$ & 35,8 \\
$2.000 €-3.000 €$ & 16,2 \\
mehr als 3.000€ & 17,3 \\
Familienstand (\%) & \\
$\quad$ Ledig & 53,5 \\
$\quad$ verheiratet/zusammenlebend & 46,5 \\
\hline Kundenbezogene Merkmale & \\
\hline Beziehungslänge (\%) & \\
$\quad$ bis zu einem Monat & 0,7 \\
bis zu einem halben Jahr & 7,5 \\
bis zu einem Jahr & 15,7 \\
bis zu 4 Jahren & 46,4 \\
bis zu 10 Jahren & 28,2 \\
bis zu 20 Jahren & 1,4 \\
$\varnothing$ Rechnungsbetrag (€) & $16,34(\mathrm{SD}=6,7)$ \\
$\varnothing$ Bestellhäufigkeit (pro Monat) & $1,86(\mathrm{SD}=1,8)$ \\
$\varnothing$ Kaufanteil (\%) & $69,15(\mathrm{SD}=31,3)$ \\
\hline
\end{tabular}

[10] Zur Überprüfung der Stabilität der Untersuchungsergebnisse haben wir auf Anregung eines anonymen Gutachters die beiden Variablen „Bestellhäufigkeit“ und der „Lieferanteil“ als mögliche Kovariablen getestet. Die Ergebnisse der Hypothesenprüfung blieben dabei identisch. 


\section{Literaturhinweise:}

Adams, J.S. (1963): Toward an Understanding of Inequity, in: Journal of Abnormal and Social Psychology, 67. Jg., Nr. 5, S. 422-436.

Bearden, W.O./Oliver, R.L. (1985): The Role of Public and Private Complaining in Satisfaction with Problem Resolution, in: The Journal of Consumer Affairs, 19. Jg., Nr. 2, S. $222-240$

Bendapudi, N./Berry, L.L. (1997): Customers’ Motivations for Maintaining Relationships with Service Providers, in: Journal of Retailing, 73. Jg., Nr. 1, S. 15-37.

Bettencourt, L.A. (1997): Customer Voluntary Performance: Customers as Partners in Service Delivery, Journal of Retailing, 73. Jg., Nr. 3, S. 383-406.

Blodgett, J.G./Granbois, D.H./Walters, R.G. (1993): The Effects of Perceived Justice on Complainants' Negative Word-of-Mouth Behavior and Repatronage Intentions, in: Journal of Retailing, 69 Jg., Nr. 4, S. 399-428.

Bodey, K./Grace, D. (2006): Segmenting Service Complainers and Non-complainers on the Basis of Consumer Characteristics, in: Journal of Services Marketing, 20. Jg., Nr. 3, S. $178-187$.

Bolfing, C.P. (1989): How do Customers express Dissatisfaction and what can Services Marketers do about it, in: Journal of Services Marketing, 3. Jg., Nr. 2, S. 5-23.

Bougie, R./Pieters, R./Zeelenberg, M. (2003): Angry Customers Don't Come Back, they Get Back: The Experience and Behavioral Implications of Anger and Dissatisfaction in Services, in: Journal of the Academy of Marketing Science, 31. Jg., Nr. 4, S. 377-393.

Bruhn, M. (1998): Schweizer Kundenbarometer - Ergebnisse einer Pilotstudie zu Kundenzufriedenheit, Kundendialog und Kundenbindung in Schweizer Unternehmen, Basel.

Davidow, M. (2003): Organizational Responses to Customer Complaints: What Works and What Doesn't, in: Journal of Service Research, 5. Jg., Nr. 3, S. 225-250.

De Matos, C.A./Henrique, J.L./Rossi, C.A.V. (2007): Service Recovery Paradox: A MetaAnalysis, in: Journal of Service Research, 10. Jg., Nr. 1, S. 60-77.

Diller, H. (1996): Kundenbindung als Marketingziel, in: Marketing - Zeitschrift für Forschung und Praxis, 18. Jg., Nr. 2, S. 81-94.

Dodds, W.B./Monroe, K.B. (1991): Effects of Price, Brand, and Store Information on Buyers' Product Evaluations, Journal of Marketing Research, 28. Jg., Nr. 3, S. 307-319.

Eschweiler, M. (2006): Externe Referenzpreise - Eine empirisch gestützte verhaltenswissenschaftliche Analyse, Wiesbaden. 
Eschweiler, M./Evanschitzky, H./Woisetschläger, D. (2007): Laborexperimente in der Marketingwissenschaft - Bestandsaufnahme und Leitfaden bei varianzanalytischen Auswertungen, Münster.

Evanschitzky, H./Wunderlich, M. (2006): An Examination of Moderator Effects in the FourStage Loyalty Model, in: Journal of Service Research, 8. Jg., Nr. 4,S. 330-345.

Fishbein, M./Ajzen, I. (1975): Belief, Attitude, Intention, and Behavior: An Introduction to Theory and Research, Reading, MA.

Fornell, C./Westbrook, R. (1979): An Exploratory Study of Assertiveness, Aggressiveness and Consumer Complaint Behavior, in: Advances in Consumer Research, 6. Jg., Nr. 1, S. $105-110$.

Ganesh, J./Arnold, M.J./Reynolds, K.E. (2000): Understanding the Customer Base of Service Providers: An Examination of the Differences Between Switchers and Stayers, in: Journal of Marketing, 64. Jg., Nr. 3, S. 65-87.

Garrett, D.E. (1999): The Effectiveness of Compensation given to Complaining Customers: Is more better? Journal of Satisfaction, Dissatisfaction and Complaining Behavior, 12. Jg., Nr. 1, S. 26-35.

Glaser, W.R. (1978): Varianzanalyse, Stuttgart: Fischer.

Gronhaug, K./Zaltmann, G. (1981): Complainers and Noncomplainers Revisited: Another Look At The Data, in: Advances in Consumer Research, 8. Jg., Nr. 1, S. 83-87.

Harris, L.C./Goode, M.M.H. (2004): The Four Stages of Loyalty and the Pivotal Role of Trust: A Study of Online Service Dynamics, in: Journal of Retailing, 80. Jg., Nr. 2, 139158.

Hirschman, A.O. (1970): Exit, Voice und Loyalty - Responses to Decline in Firms, Organizations, and Firms, London.

Hogan, J.E./Lemon, K.N./ Libai, B. (2003): What is the True Value of a Lost customer?, in: Journal of Service Research, 5. Jg., Nr. 3, S. 196-208.

Homburg, C. (2000): Entwicklungstrends der deutschsprachigen Marketingforschung, in Backhaus, K. (Hrsg.): Deutschsprachige Marketingforschung - Bestandsaufnahme und Perspektiven, Stuttgart, S. 339-360.

Homburg, C./Fürst, A. (2003): Complaint Management Excellence - Leitfaden für professionelles Beschwerdemanagement, in: Management Know How Nr. M 73, Mannheim. 
Homburg, C./Fürst, A. (2005): How Organizational Complaint Handling Drives Customer Loyalty: An Analysis of the Mechanistics and the Organic Approach, in: Journal of Marketing, 69. Jg., Nr. 3, S. 95-114.

Homburg, C./Fürst, A. (2007): Beschwerdeverhalten und Beschwerdemanagement - Eine Bestandsaufnahme der Forschung und Agenda für die Zukunft, in: DBW, 67. Jg., Nr. 1, S. 41-74.

Homburg,C./Hoyer, W./Stock-Homburg, R. (2007): How to Get Customers Back? Insights Into Customer Relationship Revival Activities, in: Journal of the Academy of Marketing Science, 35 Jg., Nr. 4, S. 461-474.

Jones, T.O./Sasser Jr., W.E. (1995): Why Satisfied Customers Defect, in: Harvard Business Review, 73. Jg., Nr. 6, S. 88-91.

Jacoby, J./Jaccard, J.J. (1981): The Sources, Meaning, and Validity of Consumer Complaint Behavior: A Psychological Analysis, in: Journal of Retailing, 57. Jg., Nr. 3, S. 4-24. Kalyanaram, G./Little, J.D.C. (1994): An Empirical Analysis of Latitude of Price Acceptance in Consumer Package Goods, in: Journal of Consumer Research, 21. Jg., Nr. 3, S. 408419.

Kau, A.K./Loh, E. (2006): The Effects of Service Recovery on Consumer Satisfaction: A Comparison between Complaints and Non-complainants, in: Journal of Services Marketing, 20. Jg., Nr. 2, S. 101-111.

Keaveney, S.M. (1995): Customer Switching Behavior in Service Industries: An Exploratory Study, in: Journal of Marketing, 59. Jg., Nr. 2, S. 71-82.

Maxham, J.G. (2001): Service recovery's influence on consumer satisfaction, positive wordof-mouth, and purchase intentions, in: Journal of Business Research, 54. Jg., Nr. 1, S. $11-24$.

Maxham, J.G./Netemeyer, R.G. (2002): A Longitudinal Study of Customer Evaluations of Multiple Service Failures and Recoveries, in: Journal of Marketing, 66. Jg., Nr. 4, S. 5771.

Mittal, V./Huppertz, J. W./Khare, A. (2008): Customer complaining: The role of tie strength and information control, in: Journal of Retailing, 84. Jg., Nr. 2, S. 195-204.

Oliver, R.L. (1997): Satisfaction-A Behavioral Perspective on the Consumer, New York.

Oliver, R.L. (1999): Whence Consumer Loyalty, in: Journal of Marketing, 63. Jg., Nr. 4, S. $33-44$.

Pick, D. (2008): Wiederaufnahme vertraglicher Geschäftsbeziehungen: Eine empirische Untersuchung der Kundenperspektive, Wiesbaden. 
Podsakoff, P.M./MacKenzie, S.B./Jeong-Yeon, L./Podsakoff, N.P. (2003): Common Method Biases in Behavioral Research: A Critical Review of the Literature and Recommended Remedies, in: Journal of Applied Psychology, 88. Jg., Nr. 5, S. 879-903.

Richins, M.L. (1983a): Negative Word-of-Mouth by Dissatisfied Consumers: A Pilot Study, in: Journal of Marketing, 47. Jg., Nr. 1, S. 68-78.

Richins, M.L. (1983b): An Analysis of Consumer Interaction Styles in the Marketplace, in: Journal of Consumer Research, 10. Jg., Nr. 1, S. 73-82.

Richins, M.L. (1987): A Multivariate Analysis of Responses to Dissatisfaction, in: Journal of the Academy of Marketing Science, 15. Jg., Nr. 3, S. 2-31.

Rutsatz, U. (2004): Kundenrückgewinnung durch Direktmarketing - Das Beispiel des Versandhandels, Wiesbaden.

Shuptrine, F.K./Wenglorz, G. (1981): Comprehensive Identification of Consumers' Marketplace Problems and what they do about them, in: Advances in Consumer Research, 8. Jg., Nr. 1, S. 687-692.

Singh, J. (1990a): A Typology of Consumer Dissatisfaction Response Styles, in: Journal of Retailing, 66. Jg., Nr. 1, S. 57-99.

Singh, J. (1990b): Voice, Exit, und Negative Word-of-Mouth Behaviors: An Investigation Across Three Service Categories, in: Journal of the Academy of Marketing Science, 18. Jg., Nr. 6, S. 1-15.

Singh, J./Panday, S. (1990): Exploring the Effects of Consumers' Dissatisfaction Level on Complaint Behaviours, in: European Journal of Marketing, 25. Jg., Nr. 9, S. 7-21.

Singh, J./Wilken, R.E. (1996): When Consumers Complain. A Path Analysis of the Key Antecedents of Consumer Complaint Responses Estimates, in: Journal of the Academy of Marketing Science, 24. Jg., Nr. 4, S. 350-365.

Sirdeshmukh, D./Singh, J./Sabol, B. (2002): Consumer Trust, Value, and Loyalty in Relational Exchanges, in: Journal of Marketing, 66. Jg., Nr. 1, S. 15-37.

Sivakumar, K./Raj, S.P. (1997): Quality Tier Competition: How Price Change Influences Brand Choice and Category Choice, in: Journal of Marketing, 61. Jg. (3), S. 71-85. Smith, A.K./Bolton, R.N. (1998): An Experimental Investigation of Service Failure and Recovery: Paradox or Peril?, in: Journal of Service Research, 1. Jg., Nr. 1, S. 65-81.

Smith, A.K./Bolton, R.N. (2002): The Effects of Customers`Emotional Response to Service Failures on their Recovery Effort Evaluations and Satisfaction Judgments, in: Journal of the Academy of Marketing Science, 30. Jg., Nr. 1, S. 5-23. 
Stauss, B./Seidel, W. (2007): Beschwerdemanagement - Unzufriedene Kunden als profitable Zielgruppe, 4. Aufl., München.

Tax, S./Brown, S.W./Chundrashekaran, M. (1998): Customer Evaluations of Service Complaint Experience: Implication for Relationship Marketing, in: Journal of Marketing, 62. Jg., Nr. 2, S. 60-76.

TARP (1979): Consumer Complaint Handling in America: Final Report, Washington D.C.

TARP (1986): Consumer Complaint Handling in America: An Update Study (Part II), Washington D.C.

Tokman, M./Davis, L.M./Lemon, K.N. (2006): The WOW factor: Creating value through win-back offers to reacquire lost customers, in: Journal of Retailing, 83 Jg., Nr. 1, S. 4764.

Voorhees, C.M./Brady, M.K. (2005): A Service Perspective on the Drivers of Complaint Intentions, in: Journal of Service Research, 8. Jg., Nr. 2, S. 192-204.

Voorhees, C.M./Brady, M.K./Horowitz, D.M. (2006): A Voice from the Silent Masses: An Exploratory and Comparative Analysis of Noncomplainers, in: Journal of the Academy of Marketing Science, 34. Jg., Nr. 4, S. 514-527.

Zeithaml, V.A./Berry, L.L./Parasuraman, A. (1996): The Behavioral Consequences of Service Quality, in: Journal of Marketing, 60. Jg., Nr. 2, S. 31-46. 